\title{
MEASURE OF DEPARTURE FROM SUM-SYMMETRY MODEL FOR SQUARE CONTINGENCY TABLES WITH ORDERED CATEGORIES
}

\section{KOUJI YAMAMOTO ${ }^{1}$, MANA AIZAWA ${ }^{2}$ and SADAO TOMIZAWA ${ }^{2}$}

${ }^{1}$ Department of Clinical Epidemiology and Biostatistics

Graduate School of Medicine

Osaka University

2-2, Yamadaoka, Suita

Osaka, 565-0871

Japan

e-mail: yamamoto-k@stat.med.osaka-u.ac.jp

${ }^{2}$ Department of Information Sciences

Faculty of Science and Technology

Tokyo University of Science

Noda, Chiba, 278-8510

Japan

e-mail: isaq01.pc@gmail.com

tomizawa@is.noda.tus.ac.jp

\begin{abstract}
For the analysis of ordinal square contingency tables, Yamamoto et al. [17]

considered the sum-symmetry model. The present paper proposes a measure to represent the degree of departure from the sum-symmetry model. The proposed measure is expressed by using the power-divergence or Patil and Taillie's
\end{abstract}

2010 Mathematics Subject Classification: 62H17.

Keywords and phrases: diversity index, Kullback-Leibler information, measure, power-divergence, sum-symmetry.

Received July 22, 2016

(C) 2016 Scientific Advances Publishers 
diversity index. The relationships between the proposed measure and the normal distribution are shown. Some biomedical data are analyzed by using the proposed measure.

\section{Introduction}

Consider an $r \times r$ square contingency table with the same ordered row and column classifications. Let $p_{i j}$ denote the probability that an observation will fall in the $i$-th row and $j$-th column of the table

$(i=1, \ldots, r ; j=1, \ldots, r)$. Bowker [2] considered the symmetry (S) model defined by

$$
p_{i j}=p_{j i} \text { for } i=1, \ldots, r ; j=1, \ldots, r .
$$

This model indicates the structure of symmetry about main diagonal of the table. See Stuart [13]; Bishop et al. ([1], Chapter 8); Caussinus [3]; and Tahata and Tomizawa [14] for the related models.

When the $\mathrm{S}$ model does not hold, we are interested in measuring the degree of departure from symmetry. Tomizawa [15] and Tomizawa et al. [16] considered measures which represent the degree of departure from the $\mathrm{S}$ model.

Yamamoto et al. [17] considered the sum-symmetry (SS) model defined by

$$
p_{1(t)}=p_{2(t)} \text { for } t=3, \ldots, 2 r-1
$$

where

$$
p_{1(t)}=\sum_{(i, j) \in R(t)} \sum_{i j}, \quad p_{2(t)}=\sum_{(i, j) \in R(t)} \sum_{j i}
$$

and

$$
R(t)=\{(i, j) \mid i+j=t, i<j\} .
$$

Let $X$ and $Y$ denote the row and column variables, respectively. Then, the SS model is also expressed as 


$$
\operatorname{Pr}(X+Y=t, X<Y)=\operatorname{Pr}(X+Y=t, X>Y) \text {, for } t=3, \ldots, 2 r-1 \text {. }
$$

Given the condition that an observation falls in one of the off-diagonal cells of the square contingency table, this model may be expressed as

$$
p_{1(t)}^{*}=p_{2(t)}^{*} \text { for } t=3, \ldots, 2 r-1,
$$

where

$$
p_{1(t)}^{*}=\frac{p_{1(t)}}{\delta}, \quad p_{2(t)}^{*}=\frac{p_{2(t)}}{\delta}, \quad \delta=\sum_{i \neq j} \sum_{i j} ;
$$

namely, as

$$
\operatorname{Pr}(X+Y=t, X<Y \mid X \neq Y)=\operatorname{Pr}(X+Y=t, X>Y \mid X \neq Y),
$$

for $t=3, \ldots, 2 r-1$. When the SS model does not hold, we are interested in measuring the degree of departure from the SS model.

The purpose of this paper is to propose a measure to represent the degree of departure from the SS model for ordinal square contingency tables. The measure would be useful for comparing the degrees of departure from the SS model in several tables.

\section{Measure of Departure from the SS Model}

\subsection{Measure}

Assume that $\left\{p_{1(t)}+p_{2(t)} \neq 0\right\}$. Let

$$
q_{t}^{*}=\frac{p_{1(t)}^{*}+p_{2(t)}^{*}}{2}, \text { for } t=3, \ldots, 2 r-1
$$

The SS model may be expressed as

$$
p_{1(t)}^{*}=p_{2(t)}^{*}=q_{t}^{*}, \text { for } t=3, \ldots, 2 r-1
$$


Consider a measure defined by

$$
\Phi_{S S}^{(\lambda)}=\frac{\lambda(\lambda+1)}{2^{\lambda}-1} I^{(\lambda)}, \text { for } \lambda>-1
$$

where

$$
I^{(\lambda)}=\frac{1}{\lambda(\lambda+1)} \sum_{t=3}^{2 r-1}\left[p_{1(t)}^{*}\left[\left(\frac{p_{1(t)}^{*}}{q_{t}^{*}}\right)^{\lambda}-1\right]+p_{2(t)}^{*}\left[\left(\frac{p_{2(t)}^{*}}{q_{t}^{*}}\right)^{\lambda}-1\right]\right],
$$

and value at $\lambda=0$ is taken to be the limit as $\lambda \rightarrow 0$. Namely,

$$
\Phi_{S S}^{(0)}=\frac{1}{\log 2} I^{(0)}
$$

where

$$
I^{(0)}=\sum_{t=3}^{2 r-1}\left[p_{1(t)}^{*} \log \left(\frac{p_{1(t)}^{*}}{q_{t}^{*}}\right)+p_{2(t)}^{*} \log \left(\frac{p_{2(t)}^{*}}{q_{t}^{*}}\right)\right] .
$$

Note that $I^{(\lambda)}$ is the power-divergence between $\left\{p_{1(t)}^{*}, p_{2(t)}^{*}\right\}$ and $\left\{q_{t}^{*}, q_{t}^{*}\right\}$, $t=3, \ldots, 2 r-1$. Especially, $I^{(0)}$ is Kullback-Leibler information. Also, note that the real value of $\lambda(>-1)$ is chosen by users. For more details of the power-divergence, see Cressie \& Read [10] and Read \& Cressie ([12], p.15).

Let

$$
p_{1(t)}^{c}=\frac{p_{1(t)}}{p_{1(t)}+p_{2(t)}}, \quad p_{2(t)}^{c}=\frac{p_{2(t)}}{p_{1(t)}+p_{2(t)}} .
$$

The SS model is further expressed as

$$
p_{1(t)}^{c}=p_{2(t)}^{c}=\frac{1}{2}, \text { for } t=3, \ldots, 2 r-1 .
$$

Therefore, $\Phi_{S S}^{(\lambda)}$ can be expressed as

$$
\Phi_{S S}^{(\lambda)}=\frac{\lambda(\lambda+1)}{2^{\lambda}-1} \sum_{t=3}^{2 r-1}\left(p_{1(t)}^{*}+p_{2(t)}^{*}\right) I_{t}^{(\lambda)}, \text { for } \lambda>-1,
$$


where

$$
I_{t}^{(\lambda)}=\frac{1}{\lambda(\lambda+1)}\left[p_{1(t)}^{c}\left[\left(\frac{p_{1(t)}^{c}}{1 / 2}\right)^{\lambda}-1\right]+p_{2(t)}^{c}\left[\left(\frac{p_{2(t)}^{c}}{1 / 2}\right)^{\lambda}-1\right]\right]
$$

and value at $\lambda=0$ is taken to be the limit as $\lambda \rightarrow 0$. Namely,

$$
\Phi_{S S}^{(0)}=\frac{1}{\log 2} \sum_{t=3}^{2 r-1}\left(p_{1(t)}^{*}+p_{2(t)}^{*}\right) I_{t}^{(0)}
$$

where

$$
I_{t}^{(0)}=p_{1(t)}^{c} \log \left(\frac{p_{1(t)}^{c}}{1 / 2}\right)+p_{2(t)}^{c} \log \left(\frac{p_{2(t)}^{c}}{1 / 2}\right)
$$

The measure $\Phi_{S S}^{(\lambda)}$ represents the weighted average of $\left\{I_{t}^{(\lambda)}\right\}$.

Furthermore, $\Phi_{S S}^{(\lambda)}$ may be expressed as

$$
\Phi_{S S}^{(\lambda)}=\sum_{t=3}^{2 r-1}\left(p_{1(t)}^{*}+p_{2(t)}^{*}\right)\left(1-\frac{\lambda 2^{\lambda}}{2^{\lambda}-1} H_{t}^{(\lambda)}\right), \text { for } \lambda>-1
$$

where

$$
H_{t}^{(\lambda)}=\frac{1}{\lambda}\left[1-\left(p_{1(t)}^{c}\right)^{\lambda+1}-\left(p_{2(t)}^{c}\right)^{\lambda+1}\right],
$$

and value at $\lambda=0$ is taken to be the limit as $\lambda \rightarrow 0$. Namely,

$$
\Phi_{S S}^{(0)}=\sum_{t=3}^{2 r-1}\left(p_{1(t)}^{*}+p_{2(t)}^{*}\right)\left(1-\frac{1}{\log 2} H_{t}^{(0)}\right)
$$

where

$$
H_{t}^{(0)}=-p_{1(t)}^{c} \log p_{1(t)}^{c}-p_{2(t)}^{c} \log p_{2(t)}^{c}
$$


Note that $H_{t}^{(\lambda)}$ is Patil and Taillie's [11] diversity index of degree- $\lambda$ for $\left\{p_{1(t)}^{c}, p_{2(t)}^{c}\right\}$, which includes the Shannon entropy (when $\lambda=0$ ) and the Gini concentration or Simpson's index (when $\lambda=1$ ) in special cases.

Noting that $I^{(\lambda)} \geq 0$ and $H_{t}^{(\lambda)} \geq 0$ for each $\lambda$, we see that $\Phi_{S S}^{(\lambda)}$ lies between 0 and 1 . For each $\lambda(>-1)$, (i) $\Phi_{S S}^{(\lambda)}=0$ if and only if there is a structure of the SS model in the table, i.e., $p_{1(t)}=p_{2(t)}$ for $t=3, \ldots, 2 r-1$, and (ii) $\Phi_{S S}^{(\lambda)}=1$ if and only if the degree of departure from the SS model is largest, i.e., $p_{1(t)}=0$ (then $p_{2(t)} \neq 0$ ) or $p_{2(t)}=0$ (then $p_{1(t)} \neq 0$ ) for $t=3, \ldots, 2 r-1$. According to the weighted sum of power-divergence or Patil and Taillie's diversity index, $\Phi_{S S}^{(\lambda)}$ represents degree of departure from the SS model, and the degree increases as the value of $\Phi_{S S}^{(\lambda)}$ increases.

\subsection{Submeasure}

When the SS model does not hold, we are interested in finding which set of cells $(i, j)$ with $i+j=t$ for fixed $t$ (for $t=3, \ldots, 2 r-1$ ) has a large degree of departure from symmetric structure.

For $t=3, \ldots, 2 r-1$, assuming that $p_{1(t)}+p_{2(t)}>0$, consider a measure defined by

$$
\begin{aligned}
\Phi_{S S(t)}^{(\lambda)} & =\frac{\lambda(\lambda+1)}{2^{\lambda}-1} I_{t}^{(\lambda)} \\
& =1-\frac{\lambda 2^{\lambda}}{2^{\lambda}-1} H_{t}^{(\lambda)}, \quad \text { for } \lambda>-1
\end{aligned}
$$

where the value at $\lambda=0$ is taken to be the limit as $\lambda \rightarrow 0$. For each $t$ and $\lambda$, we see that $\Phi_{S S(t)}^{(\lambda)}$ lies between 0 and 1 ; also, it is seen that 
(i) $\Phi_{S S(t)}^{(\lambda)}=0$ if and only if $p_{1(t)}=p_{2(t)}$, and (ii) $\Phi_{S S(t)}^{(\lambda)}=1$ if and only if $p_{1(t)}=0$ or $p_{2(t)}=0$. For fixed $t$, the degree of departure from the equality of $p_{1(t)}$ and $p_{2(t)}$ increases as the value of $\Phi_{S S(t)}^{(\lambda)}$ increases.

\section{Approximate Confidence Interval for Measure}

Let $\left\{n_{i j}\right\}$ denote the observed frequency in the $i$-th row and $j$-th column of the $r \times r$ table $(i=1, \ldots, r ; j=1, \ldots, r)$. Assuming that a multinomial distribution applies to the table, we shall consider an approximate standard error and large-sample confidence interval for $\Phi_{S S}^{(\lambda)}$ using the delta method, descriptions of which are given by Bishop et al. ([1], Subsection 14.6). Let $\hat{\Phi}_{S S}^{(\lambda)}$ denote the sample version of $\Phi_{S S}^{(\lambda)}$, namely, $\Phi_{S S}^{(\lambda)}$ with $\left\{p_{i j}\right\}$ replaced by $\left\{\hat{p}_{i j}\right\}$, where $\hat{p}_{i j}=n_{i j} / n$ and $n=\sum \sum n_{i j}$. Using the delta method, $\sqrt{n}\left(\hat{\Phi}_{S S}^{(\lambda)}-\Phi_{S S}^{(\lambda)}\right)$ has asymptotically (as $n \rightarrow \infty$ ) a normal distribution with mean 0 and variance $\sigma^{2}\left[\Phi_{S S}^{(\lambda)}\right]$. The asymptotic variance $\sigma^{2}\left[\Phi_{S S}^{(\lambda)}\right]$ for $\lambda>-1$ is expressed as follows: (i) for $\lambda \neq 0$,

$$
\sigma^{2}\left[\Phi_{S S}^{(\lambda)}\right]=\left(\frac{1}{\delta\left(2^{\lambda}-1\right)}\right)^{2} \sum_{t=3}^{2 r-1}\left[\Delta_{1(t)}^{2} p_{1(t)}+\Delta_{2(t)}^{2} p_{2(t)}\right]-\frac{1}{\delta}\left(\Phi_{S S}^{(\lambda)}\right)^{2}
$$

where

$$
\begin{aligned}
& \Delta_{1(t)}=\left(2 p_{1(t)}^{c}\right)^{\lambda}-1+\lambda p_{2(t)}^{c}\left[\left(2 p_{1(t)}^{c}\right)^{\lambda}-\left(2 p_{2(t)}^{c}\right)^{\lambda}\right], \\
& \Delta_{2(t)}=\left(2 p_{2(t)}^{c}\right)^{\lambda}-1+\lambda p_{1(t)}^{c}\left[\left(2 p_{2(t)}^{c}\right)^{\lambda}-\left(2 p_{1(t)}^{c}\right)^{\lambda}\right],
\end{aligned}
$$

and (ii) for $\lambda=0$,

$$
\sigma^{2}\left[\Phi_{S S}^{(0)}\right]=\left(\frac{1}{\delta(\log 2)}\right)^{2} \sum_{t=3}^{2 r-1}\left[\left(\log 2 p_{1(t)}^{c}\right)^{2} p_{1(t)}+\left(\log 2 p_{2(t)}^{c}\right)^{2} p_{2(t)}\right]-\frac{1}{\delta}\left(\Phi_{S S}^{(0)}\right)^{2}
$$


Let $\hat{\sigma}^{2}\left[\Phi_{S S}^{(\lambda)}\right]$ denote $\sigma^{2}\left[\Phi_{S S}^{(\lambda)}\right]$ with $\left\{p_{i j}\right\}$ replaced by $\left\{\hat{p}_{i j}\right\}$. Then, $\hat{\sigma}\left[\Phi_{S S}^{(\lambda)}\right] / \sqrt{n}$ is an estimated standard error for $\hat{\Phi}_{S S}^{(\lambda)}$, and $\hat{\Phi}_{S S}^{(\lambda)} \pm z_{\alpha / 2} \hat{\sigma}\left[\Phi_{S S}^{(\lambda)}\right] / \sqrt{n}$ is an approximate $100(1-\alpha) \%$ confidence interval for $\Phi_{S S}^{(\lambda)}$, where $z_{\alpha / 2}$ is the upper $100 \times(\alpha / 2)$ percentage point from the standard normal distribution.

Similarly, the approximate confidence interval for $\Phi_{S S(t)}^{(\lambda)}$ is obtained by using the asymptotic variance $\hat{\sigma}^{2}\left[\Phi_{S S(t)}^{(\lambda)}\right]$, where (i) for $\lambda \neq 0$,

$$
\sigma^{2}\left[\Phi_{S S(t)}^{(\lambda)}\right]=\left(\frac{1+\lambda}{2^{\lambda}-1}\right)^{2} \frac{p_{1(t)}^{c} p_{2(t)}^{c}}{p_{1(t)}+p_{2(t)}}\left[\left(2 p_{1(t)}^{c}\right)^{\lambda}-\left(2 p_{2(t)}^{c}\right)^{\lambda}\right]^{2},
$$

and (ii) for $\lambda=0$,

$$
\sigma^{2}\left[\Phi_{S S(t)}^{(0)}\right]=\left(\frac{1}{\log 2}\right)^{2} \frac{p_{1(t)}^{c} p_{2(t)}^{c}}{p_{1(t)}+p_{2(t)}}\left(\log p_{1(t)}^{c}-\log p_{2(t)}^{c}\right)^{2}
$$

\section{Examples}

\subsection{Example 1}

Consider the data in Tables 1(a) and 1(b). Table 1(a) is the data of hearing threshold at $6000 \mathrm{~Hz}$ in decibels of 1664 examinees aged 20-69 in National Health and Nutrition Examination Survey [4]. Table 1(b) is the data of hearing threshold at $8000 \mathrm{~Hz}$ in decibels of 1751 examinees aged 20-69 in National Health and Nutrition Examination Survey [5]. In Tables 1(a) and 1(b), the row variable is the right ear threshold grade and column value is the left ear threshold grade. The category in both Tables 1(a) and 1(b) means thresholds of hearing impairment severity based on National Center for Health Statistics, Audiometry/Tympanometry Procedures Manual [6] : (1) is $\leq 25 \mathrm{~dB}$ (i.e., normal hearing), (2) is $26-40 \mathrm{~dB}$ (i.e., mild hearing loss), (3) is $41-55 \mathrm{~dB}$ (i.e., moderate hearing loss), and (4) is $56+d B$ (i.e., moderately severe or greater hearing loss). 
Table 1. Hearing threshold in decibels of examinees aged 20-69, National Health and Nutrition Examination Survey [4, 5]

\begin{tabular}{cccccc}
\hline Right ear & \multicolumn{4}{c}{ Left ear threshold grade } \\
\cline { 2 - 5 } threshold grade & $(1)$ & $(2)$ & (3) & (4) & Total \\
\hline
\end{tabular}

(a) Threshold at $6000 \mathrm{~Hz}$, examined in 1999-2000

\begin{tabular}{cccccc}
$(1)$ & 913 & 153 & 30 & 25 & 1121 \\
$(2)$ & 87 & 101 & 53 & 21 & 262 \\
$(3)$ & 11 & 33 & 45 & 34 & 123 \\
$(4)$ & 7 & 14 & 23 & 114 & 158 \\
\hline Total & 1018 & 301 & 151 & 194 & 1664 \\
\hline
\end{tabular}

(b) Threshold at $8000 \mathrm{~Hz}$, examined in 2003-2004

\begin{tabular}{cccccc}
$(1)$ & 1059 & 86 & 23 & 12 & 1180 \\
$(2)$ & 95 & 80 & 35 & 20 & 230 \\
$(3)$ & 22 & 35 & 50 & 34 & 141 \\
$(4)$ & 9 & 18 & 42 & 131 & 200 \\
\hline Total & 1185 & 219 & 150 & 197 & 1751 \\
\hline
\end{tabular}

(1) $\leq 25 \mathrm{~dB}$, (2) $26-40 \mathrm{~dB}$, (3) 41-55dB, and (4) 56+ dB.

It seems natural to see the degree of an individual's hearing threshold grade as the sum of the grades of both right and left ears, and therefore it would be meaningful to apply the SS model for these data.

Since the confidence interval for $\Phi_{S S}^{(\lambda)}$ applied to the data in Table 1(a) does not include zero for all $\lambda$ (Table 2), this would indicate that there is not a structure of the SS model in Table 1(a). In contrast, including zero, the confidence interval for $\Phi_{S S}^{(\lambda)}$ applied to the data in Table 1(b) would indicate that there is a structure of the SS model in Table 1(b). 
Table 2. Estimate of $\Phi_{S S}^{(\lambda)}$, estimated approximate standard error for $\hat{\Phi}_{S S}^{(\lambda)}$ and approximate $95 \%$ confidence interval for $\Phi_{S S}^{(\lambda)}$, applied to Tables 1(a) and 1(b)

\begin{tabular}{|c|c|c|c|}
\hline$\lambda$ & $\begin{array}{c}\text { Estimated } \\
\text { measure }\end{array}$ & $\begin{array}{c}\text { Standard } \\
\text { error }\end{array}$ & $\begin{array}{c}\text { Confidence } \\
\text { interval }\end{array}$ \\
\hline \multicolumn{4}{|c|}{ (a) For Table 1(a) } \\
\hline-0.4 & 0.045 & 0.013 & $(0.018,0.071)$ \\
\hline 0.0 & 0.064 & 0.019 & $(0.027,0.101)$ \\
\hline 0.6 & 0.081 & 0.024 & $(0.035,0.128)$ \\
\hline 1.0 & 0.087 & 0.025 & $(0.038,0.136)$ \\
\hline 1.6 & 0.089 & 0.026 & $(0.039,0.139)$ \\
\hline 2.0 & 0.087 & 0.025 & $(0.038,0.136)$ \\
\hline \multicolumn{4}{|c|}{ (b) For Table 1(b) } \\
\hline-0.4 & 0.002 & 0.003 & $(-0.004,0.007)$ \\
\hline 0.0 & 0.003 & 0.004 & $(-0.006,0.011)$ \\
\hline 0.6 & 0.003 & 0.005 & $(-0.007,0.014)$ \\
\hline 1.0 & 0.004 & 0.006 & $(-0.008,0.015)$ \\
\hline 1.6 & 0.004 & 0.006 & $(-0.008,0.015)$ \\
\hline 2.0 & 0.004 & 0.006 & $(-0.008,0.015)$ \\
\hline
\end{tabular}

Comparing Table 1(a) with Table 1(b) by using confidence interval for $\Phi_{S S}^{(\lambda)}$, we can see that the degree of departure from the SS model is greater in Table 1(a) than in Table 1(b).

We shall further analyze the data in Tables 1(a) and 1(b) using submeasures $\Phi_{S S(t)}^{(\lambda)}$ for $t=3, \ldots, 7$. We see from Table 3 that for Table 1(a), the confidence intervals for $\Phi_{S S(4)}^{(\lambda)}, \Phi_{S S(5)}^{(\lambda)}, \Phi_{S S(6)}^{(\lambda)}$, and $\Phi_{S S(7)}^{(\lambda)}$ include zero (for $\lambda>-1$ ), but the confidence interval for $\Phi_{S S(3)}^{(\lambda)}$ does not include zero (for $\lambda>-1$ ). Thus, this would indicate that (i) the probability that the degree of the hearing threshold grade for an individual whose left ear threshold grade is greater than his/her right ear 
threshold, is $t(t=4, \ldots, 7)$, is equal to the probability that the degree of it whose right ear threshold grade is greater than his/her left ear threshold grade, is $t$, but (ii) the probability that the degree of the hearing threshold grade for an individual whose left ear threshold grade is greater than his/her right ear threshold, is 3 , is not equal to the probability that the degree of it whose right ear threshold grade is greater than his/her left ear threshold grade, is 3 . Thus, we can guess that the SS structure with $t=3$ may influence on the lack of the structure of the SS model. 
KOUJI YAMAMOTO et al.

Table 3. Estimate of submeasure applied to Table 1(a)

\begin{tabular}{|c|c|c|c|c|}
\hline & $\lambda$ & $\begin{array}{c}\text { Estimated } \\
\text { measure }\end{array}$ & $\begin{array}{c}\text { Standard } \\
\text { error }\end{array}$ & $\begin{array}{c}\text { Confidence } \\
\text { interval }\end{array}$ \\
\hline \multirow[t]{6}{*}{$\Phi_{S S(3)}^{(\lambda)}$} & -0.4 & 0.038 & 0.018 & $(0.004,0.073)$ \\
\hline & 0.0 & 0.055 & 0.025 & $(0.006,0.105)$ \\
\hline & 0.6 & 0.071 & 0.032 & $(0.008,0.133)$ \\
\hline & 1.0 & 0.076 & 0.034 & $(0.009,0.143)$ \\
\hline & 1.6 & 0.077 & 0.035 & $(0.009,0.146)$ \\
\hline & 2.0 & 0.076 & 0.034 & $(0.009,0.143)$ \\
\hline \multirow[t]{6}{*}{$\Phi_{S S(4)}^{(\lambda)}$} & -0.4 & 0.114 & 0.073 & $(-0.029,0.256)$ \\
\hline & 0.0 & 0.161 & 0.100 & $(-0.035,0.357)$ \\
\hline & 0.6 & 0.202 & 0.122 & $(-0.037,0.441)$ \\
\hline & 1.0 & 0.215 & 0.128 & $(-0.037,0.466)$ \\
\hline & 1.6 & 0.219 & 0.130 & $(-0.036,0.474)$ \\
\hline & 2.0 & 0.215 & 0.128 & $(-0.037,0.466)$ \\
\hline \multirow[t]{6}{*}{$\Phi_{S S(5)}^{(\lambda)}$} & -0.4 & 0.053 & 0.030 & $(-0.005,0.111)$ \\
\hline & 0.0 & 0.076 & 0.042 & $(-0.006,0.158)$ \\
\hline & 0.6 & 0.097 & 0.053 & $(-0.006,0.200)$ \\
\hline & 1.0 & 0.104 & 0.056 & $(-0.006,0.214)$ \\
\hline & 1.6 & 0.106 & 0.057 & $(-0.006,0.218)$ \\
\hline & 2.0 & 0.104 & 0.056 & $(-0.006,0.214)$ \\
\hline \multirow[t]{6}{*}{$\Phi_{S S(6)}^{(\lambda)}$} & -0.4 & 0.020 & 0.034 & $(-0.046,0.086)$ \\
\hline & 0.0 & 0.029 & 0.048 & $(-0.066,0.124)$ \\
\hline & 0.6 & 0.037 & 0.062 & $(-0.084,0.159)$ \\
\hline & 1.0 & 0.040 & 0.066 & $(-0.090,0.170)$ \\
\hline & 1.6 & 0.041 & 0.068 & $(-0.092,0.174)$ \\
\hline & 2.0 & 0.040 & 0.066 & $(-0.090,0.170)$ \\
\hline
\end{tabular}


Table 3. (Continued)

\begin{tabular}{lrlll}
\hline$\Phi_{S S(7)}^{(\lambda)}$ & -0.4 & 0.019 & 0.025 & $(-0.031,0.068)$ \\
& 0.0 & 0.027 & 0.037 & $(-0.045,0.099)$ \\
& 0.6 & 0.035 & 0.047 & $(-0.057,0.127)$ \\
1.0 & 0.037 & 0.050 & $(-0.061,0.136)$ \\
& 1.6 & 0.038 & 0.051 & $(-0.062,0.139)$ \\
& 2.0 & 0.037 & 0.050 & $(-0.061,0.136)$ \\
\hline
\end{tabular}

In contrast, from Table 4 , the confidence intervals for $\Phi_{S S(t)}^{(\lambda)}$ $(t=3, \ldots, 7)$ of data in Table 1(b) include zero (for $\lambda>-1)$. Thus, this would indicate that, for Table 1(b), the probability that the degree of the hearing threshold grade for an individual whose left ear threshold grade is greater than his/her right ear threshold, is $t(t=3, \ldots, 7)$, is equal to the probability that the degree of it whose right ear threshold grade is greater than his/her left ear threshold grade, is $t$. 
KOUJI YAMAMOTO et al.

Table 4. Estimate of submeasure applied to Table 1(b)

\begin{tabular}{|c|c|c|c|c|}
\hline & $\lambda$ & $\begin{array}{c}\text { Estimated } \\
\text { measure }\end{array}$ & $\begin{array}{c}\text { Standard } \\
\text { error }\end{array}$ & $\begin{array}{c}\text { Confidence } \\
\text { interval }\end{array}$ \\
\hline \multirow[t]{6}{*}{$\Phi_{S S(3)}^{(\lambda)}$} & -0.4 & 0.001 & 0.004 & $(-0.006,0.008)$ \\
\hline & 0.0 & 0.002 & 0.005 & $(-0.009,0.012)$ \\
\hline & 0.6 & 0.002 & 0.007 & $(-0.011,0.016)$ \\
\hline & 1.0 & 0.002 & 0.007 & $(-0.012,0.017)$ \\
\hline & 1.6 & 0.003 & 0.008 & $(-0.012,0.017)$ \\
\hline & 2.0 & 0.002 & 0.007 & $(-0.012,0.017)$ \\
\hline \multirow[t]{6}{*}{$\Phi_{S S(4)}^{(\lambda)}$} & -0.4 & 0.000 & 0.003 & $(-0.006,0.007)$ \\
\hline & 0.0 & 0.000 & 0.005 & $(-0.009,0.010)$ \\
\hline & 0.6 & 0.000 & 0.006 & $(-0.012,0.013)$ \\
\hline & 1.0 & 0.000 & 0.007 & $(-0.012,0.013)$ \\
\hline & 1.6 & 0.001 & 0.007 & $(-0.013,0.014)$ \\
\hline & 2.0 & 0.000 & 0.007 & $(-0.012,0.013)$ \\
\hline \multirow[t]{6}{*}{$\Phi_{S S(5)}^{(\lambda)}$} & -0.4 & 0.001 & 0.003 & $(-0.006,0.007)$ \\
\hline & 0.0 & 0.001 & 0.005 & $(-0.009,0.011)$ \\
\hline & 0.6 & 0.001 & 0.006 & $(-0.012,0.014)$ \\
\hline & 1.0 & 0.001 & 0.007 & $(-0.012,0.015)$ \\
\hline & 1.6 & 0.001 & 0.007 & $(-0.013,0.015)$ \\
\hline & 2.0 & 0.001 & 0.007 & $(-0.012,0.015)$ \\
\hline \multirow[t]{6}{*}{$\Phi_{S S(6)}^{(\lambda)}$} & -0.4 & 0.001 & 0.008 & $(-0.015,0.018)$ \\
\hline & 0.0 & 0.002 & 0.012 & $(-0.022,0.026)$ \\
\hline & 0.6 & 0.003 & 0.016 & $(-0.029,0.034)$ \\
\hline & 1.0 & 0.003 & 0.017 & $(-0.031,0.036)$ \\
\hline & 1.6 & 0.003 & 0.017 & $(-0.031,0.037)$ \\
\hline & 2.0 & 0.003 & 0.017 & $(-0.031,0.036)$ \\
\hline
\end{tabular}


Table 4. (Continued)

\begin{tabular}{lcccc}
\hline$\Phi_{S S(7)}^{(\lambda)}$ & -0.4 & 0.006 & 0.012 & $(-0.018,0.029)$ \\
& 0.0 & 0.008 & 0.017 & $(-0.026,0.042)$ \\
& 0.6 & 0.010 & 0.022 & $(-0.034,0.054)$ \\
1.0 & 0.011 & 0.024 & $(-0.036,0.058)$ \\
1.6 & 0.011 & 0.025 & $(-0.037,0.060)$ \\
& 2.0 & 0.011 & 0.024 & $(-0.036,0.058)$ \\
\hline
\end{tabular}

\subsection{Example 2}

Consider the data in Tables 5(a) and 5(b). Table 5(a) is the data of grip strength of 2356 men aged 15-69 in National Health and Nutrition Examination Survey [8]. Table 5(b) is the data of grip strength of 2308 women aged 15-69 in National Health and Nutrition Examination Survey [8]. In Tables 5(a) and 5(b), the row variable is the right hand muscle strength level and column variable is the left hand muscle strength level. The category in both Tables 5(a) and 5(b) means muscle strength level compared with other people of one's age and sex. This classification is based on National Center for Health Statistics, Muscle Strength Procedure Manual [7]. 
Table 5. Grip strength test of examinees aged 15-69, National Health and Nutrition Examination Survey [8]

\begin{tabular}{ccccccc}
\hline \multirow{2}{*}{$\begin{array}{c}\text { Right hand } \\
\text { grip strength level }\end{array}$} & \multicolumn{5}{c}{ Left hand grip strength level } \\
\cline { 3 - 5 } (a) Men & $(2)$ & $(3)$ & $(4)$ & $(5)$ & Total \\
$(1)$ & 215 & 124 & 46 & 14 & 2 & 401 \\
$(2)$ & 37 & 143 & 165 & 74 & 16 & 435 \\
$(3)$ & 7 & 45 & 156 & 166 & 51 & 425 \\
$(4)$ & 2 & 20 & 62 & 226 & 210 & 520 \\
$(5)$ & 1 & 2 & 16 & 61 & 495 & 575 \\
\hline Total & 262 & 334 & 445 & 541 & 774 & 2356 \\
\hline
\end{tabular}

(b) Women

\begin{tabular}{ccccccc}
$(1)$ & 380 & 229 & 49 & 17 & 2 & 677 \\
$(2)$ & 34 & 204 & 198 & 80 & 23 & 539 \\
$(3)$ & 5 & 55 & 159 & 161 & 74 & 454 \\
$(4)$ & 1 & 11 & 37 & 106 & 168 & 323 \\
$(5)$ & 0 & 2 & 12 & 45 & 256 & 315 \\
\hline Total & 420 & 501 & 455 & 409 & 523 & 2308 \\
\hline
\end{tabular}

(1) Excellent, (2) Very good, (3) Good, (4) Fair, and (5) Poor.

It seems natural to see the degree of an individual's muscle strength level as the sum of the levels of both right and left hands, and therefore it would be meaningful to apply the SS model for these data.

Since the confidence intervals for $\Phi_{S S}^{(\lambda)}$ applied to the data in Tables 5 (a) and 5(b) do not include zero for all $\lambda$ (Table 6), this would indicate that there is not a structure of the SS model in each of Tables 5(a) and 5(b). 
Table 6. Estimate of $\Phi_{S S}^{(\lambda)}$, estimated approximate standard error for $\hat{\Phi}_{S S}^{(\lambda)}$ and approximate $95 \%$ confidence interval for $\Phi_{S S}^{(\lambda)}$, applied to Tables 5(a) and 5(b)

\begin{tabular}{cccc}
\hline$\lambda$ & Estimated & $\begin{array}{c}\text { Standard } \\
\text { error }\end{array}$ & $\begin{array}{c}\text { Confidence } \\
\text { interval }\end{array}$ \\
\hline (a) For Table 5(a) & & \\
-0.4 & 0.167 & 0.017 & $(0.134,0.200)$ \\
0.0 & 0.233 & 0.022 & $(0.189,0.277)$ \\
0.6 & 0.288 & 0.026 & $(0.236,0.339)$ \\
1.0 & 0.304 & 0.027 & $(0.251,0.357)$ \\
1.6 & 0.309 & 0.028 & $(0.255,0.363)$ \\
2.0 & 0.304 & 0.027 & $(0.251,0.357)$ \\
\hline (b) For Table $5(\mathrm{~b})$ & & \\
-0.4 & 0.262 & 0.020 & $(0.223,0.301)$ \\
0.0 & 0.355 & 0.025 & $(0.306,0.404)$ \\
0.6 & 0.427 & 0.028 & $(0.373,0.481)$ \\
1.0 & 0.447 & 0.028 & $(0.392,0.502)$ \\
1.6 & 0.453 & 0.028 & $(0.398,0.509)$ \\
2.0 & 0.447 & 0.028 & $(0.392,0.502)$ \\
\hline
\end{tabular}

Comparing Table 5(a) with Table 5(b) by using confidence interval for $\Phi_{S S}^{(\lambda)}$, we can see that the degree of departure from the SS model is greater in Table 5(b) than in Table 5(a).

We shall further analyze the data in Tables 5(a) and 5(b) using submeasures $\Phi_{S S(t)}^{(\lambda)}$ for $t=3, \ldots, 9$. We see from Table 7 that for the data in Table 5(a), $\hat{\Phi}_{S S(4)}^{(\lambda)}$ is largest for each $\lambda$. Thus, this would indicate that the probability that the degree of the muscle strength level for an individual whose left hand strength level is greater than his right hand strength level, is 4 , and probability that the degree of it whose right hand strength level is greater than his left hand strength level, is 4, has a large degree of departure from symmetric structure. We note from Table 8 that we can obtain a similar interpretation for the data in Table 5(b). 
KOUJI YAMAMOTO et al.

Table 7. Estimate of submeasure applied to Table 5(a)

\begin{tabular}{|c|c|c|c|c|}
\hline & $\lambda$ & $\begin{array}{l}\text { Estimated } \\
\text { measure }\end{array}$ & $\begin{array}{c}\text { Standard } \\
\text { error }\end{array}$ & $\begin{array}{c}\text { Confidence } \\
\text { interval }\end{array}$ \\
\hline \multirow[t]{6}{*}{$\Phi_{S S(3)}^{(\lambda)}$} & -0.4 & 0.159 & 0.043 & $(0.074,0.243)$ \\
\hline & 0.0 & 0.222 & 0.058 & $(0.109,0.336)$ \\
\hline & 0.6 & 0.276 & 0.069 & $(0.141,0.411)$ \\
\hline & 1.0 & 0.292 & 0.072 & $(0.152,0.432)$ \\
\hline & 1.6 & 0.297 & 0.072 & $(0.155,0.439)$ \\
\hline & 2.0 & 0.292 & 0.072 & $(0.152,0.432)$ \\
\hline \multirow[t]{6}{*}{$\Phi_{S S(4)}^{(\lambda)}$} & -0.4 & 0.326 & 0.104 & $(0.122,0.530)$ \\
\hline & 0.0 & 0.437 & 0.126 & $(0.189,0.684)$ \\
\hline & 0.6 & 0.519 & 0.136 & $(0.253,0.786)$ \\
\hline & 1.0 & 0.541 & 0.137 & $(0.273,0.810)$ \\
\hline & 1.6 & 0.548 & 0.137 & $(0.280,0.816)$ \\
\hline & 2.0 & 0.541 & 0.137 & $(0.273,0.810)$ \\
\hline \multirow[t]{6}{*}{$\Phi_{S S(5)}^{(\lambda)}$} & -0.4 & 0.189 & 0.039 & $(0.112,0.266)$ \\
\hline & 0.0 & 0.262 & 0.052 & $(0.160,0.365)$ \\
\hline & 0.6 & 0.323 & 0.061 & $(0.204,0.442)$ \\
\hline & 1.0 & 0.341 & 0.063 & $(0.218,0.465)$ \\
\hline & 1.6 & 0.347 & 0.064 & $(0.222,0.472)$ \\
\hline & 2.0 & 0.341 & 0.063 & $(0.218,0.465)$ \\
\hline \multirow[t]{6}{*}{$\Phi_{S S(6)}^{(\lambda)}$} & -0.4 & 0.177 & 0.058 & $(0.062,0.291)$ \\
\hline & 0.0 & 0.246 & 0.078 & $(0.094,0.398)$ \\
\hline & 0.6 & 0.304 & 0.091 & $(0.125,0.483)$ \\
\hline & 1.0 & 0.322 & 0.095 & $(0.136,0.507)$ \\
\hline & 1.6 & 0.327 & 0.096 & $(0.139,0.515)$ \\
\hline & 2.0 & 0.322 & 0.095 & $(0.136,0.507)$ \\
\hline
\end{tabular}


MEASURE OF DEPARTURE FROM SUM-SYMMETRY ...

Table 7. (Continued)

\begin{tabular}{|c|c|c|c|c|}
\hline \multirow[t]{6}{*}{$\Phi_{S S(7)}^{(\lambda)}$} & -0.4 & 0.122 & 0.031 & $(0.062,0.183)$ \\
\hline & 0.0 & 0.173 & 0.042 & $(0.090,0.256)$ \\
\hline & 0.6 & 0217 & 0.051 & $(0.116,0.317)$ \\
\hline & 1.0 & 0.230 & 0.054 & $(0.125,0.335)$ \\
\hline & 1.6 & 0.234 & 0.054 & $(0.128,0.341)$ \\
\hline & 2.0 & 0.230 & 0.054 & $(0.125,0.335)$ \\
\hline \multirow[t]{6}{*}{$\Phi_{S S(8)}^{(\lambda)}$} & -0.4 & 0.147 & 0.064 & $(0.021,0.273)$ \\
\hline & 0.0 & 0.207 & 0.087 & $(0.036,0.378)$ \\
\hline & 0.6 & 0.257 & 0.104 & $(0.053,0.462)$ \\
\hline & 1.0 & 0.273 & 0.109 & $(0.060,0.486)$ \\
\hline & 1.6 & 0.278 & 0.110 & $(0.062,0.494)$ \\
\hline & 2.0 & 0.273 & 0.109 & $(0.060,0.486)$ \\
\hline \multirow[t]{6}{*}{$\Phi_{S S(9)}^{(\lambda)}$} & -0.4 & 0.165 & 0.034 & $(0.099,0.231)$ \\
\hline & 0.0 & 0.231 & 0.045 & $(0.142,0.319)$ \\
\hline & 0.6 & 0.286 & 0.054 & $(0.181,0.391)$ \\
\hline & 1.0 & 0.302 & 0056 & $(0.193,0.412)$ \\
\hline & 1.6 & 0.308 & 0.056 & $(0.197,0.418)$ \\
\hline & 2.0 & 0.302 & 0.056 & $(0.193,0.412)$ \\
\hline
\end{tabular}


KOUJI YAMAMOTO et al.

Table 8. Estimate of submeasure applied to Table 5(b)

\begin{tabular}{|c|c|c|c|c|}
\hline & $\lambda$ & $\begin{array}{l}\text { Estimated } \\
\text { measure }\end{array}$ & $\begin{array}{c}\text { Standard } \\
\text { error }\end{array}$ & $\begin{array}{c}\text { Confidence } \\
\text { interval }\end{array}$ \\
\hline \multirow[t]{6}{*}{$\Phi_{S S(3)}^{(\lambda)}$} & -0.4 & 0.332 & 0.047 & $(0.240,0.424)$ \\
\hline & 0.0 & 0.445 & 0.057 & $(0.333,0.556)$ \\
\hline & 0.6 & 0.527 & 0.061 & $(0.408,0.647)$ \\
\hline & 1.0 & 0.550 & 0.061 & $(0.429,0.670)$ \\
\hline & 1.6 & 0.556 & 0.061 & $(0.436,0.676)$ \\
\hline & 2.0 & 0.550 & 0.061 & $(0.429,0.670)$ \\
\hline \multirow[t]{6}{*}{$\Phi_{S S(4)}^{(\lambda)}$} & -0.4 & 0.427 & 0.115 & $(0.201,0.652)$ \\
\hline & 0.0 & 0.555 & 0.130 & $(0.300,0.810)$ \\
\hline & 0.6 & 0.642 & 0.130 & $(0.386,0.898)$ \\
\hline & 1.0 & 0.664 & 0.129 & $(0.412,0.916)$ \\
\hline & 1.6 & 0.670 & 0.128 & $(0.420,0.920)$ \\
\hline & 2.0 & 0.664 & 0.129 & $(0.412,0.916)$ \\
\hline \multirow[t]{6}{*}{$\Phi_{S S(5)}^{(\lambda)}$} & -0.4 & 0.191 & 0.036 & $(0.120,0.261)$ \\
\hline & 0.0 & 0.265 & 0.048 & $(0.171,0.359)$ \\
\hline & 0.6 & 0.326 & 0.056 & $(0.217,0.435)$ \\
\hline & 1.0 & 0.344 & 0.058 & $(0.231,0.457)$ \\
\hline & 1.6 & 0.350 & 0.058 & $(0.236,0.464)$ \\
\hline & 2.0 & 0.344 & 0.058 & $(0.231,0.457)$ \\
\hline \multirow[t]{6}{*}{$\Phi_{S S(6)}^{(\lambda)}$} & -0.4 & 0.358 & 0.082 & $(0.198,0.518)$ \\
\hline & 0.0 & 0.476 & 0.097 & $(0.285,0.666)$ \\
\hline & 0.6 & 0.560 & 0.102 & $(0.360,0.761)$ \\
\hline & 1.0 & 0.583 & 0.102 & $(0.382,0.783)$ \\
\hline & 1.6 & 0.589 & 0.102 & $(0.389,0.789)$ \\
\hline & 2.0 & 0.583 & 0.102 & $(0.382,0.783)$ \\
\hline
\end{tabular}


Table 8. (Continued)

\begin{tabular}{|c|c|c|c|c|}
\hline \multirow[t]{6}{*}{$\Phi_{S S(7)}^{(\lambda)}$} & -0.4 & 0.242 & 0.044 & $(0.155,0.328)$ \\
\hline & 0.0 & 0.331 & 0.057 & $(0.220,0.443)$ \\
\hline & 0.6 & 0.402 & 0.065 & $(0.276,0.529)$ \\
\hline & 1.0 & 0.423 & 0.066 & $(0.293,0.552)$ \\
\hline & 1.6 & 0.429 & 0.067 & $(0.299,0.559)$ \\
\hline & 2.0 & 0.423 & 0.066 & $(0.293,0.552)$ \\
\hline \multirow[t]{6}{*}{$\Phi_{S S(8)}^{(\lambda)}$} & -0.4 & 0.310 & 0.080 & $(0.153,0.466)$ \\
\hline & 0.0 & 0.417 & 0.098 & $(0.225,0.609)$ \\
\hline & 0.6 & 0.498 & 0.107 & $(0.289,0.707)$ \\
\hline & 1.0 & 0.520 & 0.108 & $(0.309,0.731)$ \\
\hline & 1.6 & 0.526 & 0.108 & $(0.315,0.738)$ \\
\hline & 2.0 & 0.520 & 0.108 & $(0.309,0.731)$ \\
\hline \multirow[t]{6}{*}{$\Phi_{S S(9)}^{(\lambda)}$} & -0.4 & 0.184 & 0.040 & $(0.105,0.262)$ \\
\hline & 0.0 & 0.256 & 0.053 & $(0.152,0.360)$ \\
\hline & 0.6 & 0.316 & 0.062 & $(0.194,0.438)$ \\
\hline & 1.0 & 0.333 & 0.065 & $(0.207,0.460)$ \\
\hline & 1.6 & 0.339 & 0.065 & $(0.211,0.467)$ \\
\hline & 2.0 & 0.333 & 0.065 & $(0.207,0.460)$ \\
\hline
\end{tabular}

\section{Simulation Studies}

Consider random variables $Z_{1}$ and $Z_{2}$ having a joint bivariate normal distribution with means $E\left(Z_{1}\right)=\mu_{1}$ and $E\left(Z_{2}\right)=\mu_{2}$, variance $\operatorname{Var}\left(Z_{1}\right)=\sigma_{1}^{2}$ and $\operatorname{Var}\left(Z_{2}\right)=\sigma_{2}^{2}$ with $\sigma_{1}^{2}=\sigma_{2}^{2}\left(=\sigma^{2}\right)$, and correlation $\operatorname{Corr}\left(Z_{1}, Z_{2}\right)=\rho$. Suppose that there is an underlying bivariate normal distribution with some conditions, and suppose that $4 \times 4$ table is formed by using cut-points for each variable at $\mu_{1}, \mu_{1} \pm 0.6 \sigma_{1}$. Then, in terms of simulation studies, each subtable of Table 9 gives a $4 \times 4$ table of size 10,000 , formed from an underlying bivariate normal distribution with the 
conditions $\mu_{2}-\mu_{1}=0.6, \sigma_{1}^{2}=\sigma_{2}^{2}$ and fixed $\rho(\rho=0,0.3,0.6,0.9)$. Similarly, each subtable of Table 10 gives a $4 \times 4$ table of size 10,000, formed from an underlying bivariate normal distribution with the conditions $\mu_{2}-\mu_{1}=0.4, \sigma_{1}^{2}=\sigma_{2}^{2}$ and fixed $\rho(\rho=0,0.3,0.6,0.9)$. Tables 11(a) and 11(b) give the values of $\hat{\Phi}_{S S}^{(\lambda)}$ for Tables 9 and 10, respectively.

Table 9. The $4 \times 4$ tables of sample size 10000, formed by cut-points for each variable at $\mu_{1}, \mu_{1} \pm 0.6 \sigma$, from an underlying bivariate normal distribution with the conditions $\mu_{2}-\mu_{1}=0.6, \sigma_{1}^{2}=\sigma_{2}^{2}$ and $\rho=0,0.3,0.6,0.9$

(a) $\rho=0$

\begin{tabular}{llll}
\hline 313 & 415 & 657 & 1279 \\
259 & 338 & 525 & 1151 \\
258 & 357 & 558 & 1097 \\
363 & 446 & 579 & 1405 \\
\hline
\end{tabular}

(c) $\rho=0.6$

\begin{tabular}{cccc}
\hline 846 & 716 & 645 & 570 \\
238 & 456 & 662 & 908 \\
83 & 266 & 549 & 1281 \\
32 & 126 & 389 & 2233 \\
\hline
\end{tabular}

(b) $\rho=0.3$

\begin{tabular}{cccc}
\hline 580 & 548 & 640 & 982 \\
309 & 408 & 572 & 996 \\
177 & 327 & 511 & 1206 \\
136 & 284 & 531 & 1793 \\
\hline
\end{tabular}

(d) $\rho=0.9$

\begin{tabular}{cccc}
\hline 1158 & 1011 & 510 & 84 \\
28 & 487 & 1110 & 659 \\
2 & 72 & 569 & 1526 \\
0 & 5 & 64 & 2715 \\
\hline
\end{tabular}


Table 10. The $4 \times 4$ tables of sample size 10000 , formed by cut-points for each variable at $\mu_{1}, \mu_{1} \pm 0.6 \sigma$, from an underlying bivariate normal distribution with the conditions $\mu_{2}-\mu_{1}=0.4, \sigma_{1}^{2}=\sigma_{2}^{2}$ and $\rho=0,0.3,0.6,0.9$

(a) $\rho=0$

\begin{tabular}{cccc}
\hline 424 & 497 & 663 & 1080 \\
346 & 417 & 546 & 964 \\
355 & 434 & 546 & 935 \\
482 & 515 & 622 & 1174 \\
\hline
\end{tabular}

(c) $\rho=0.6$

\begin{tabular}{cccc}
\hline 1075 & 712 & 584 & 406 \\
360 & 548 & 673 & 683 \\
149 & 350 & 601 & 1079 \\
55 & 208 & 477 & 2040 \\
\hline
\end{tabular}

(b) $\rho=0.3$

\begin{tabular}{cccc}
\hline 747 & 583 & 642 & 778 \\
427 & 477 & 574 & 807 \\
274 & 385 & 531 & 1031 \\
197 & 367 & 581 & 1599 \\
\hline
\end{tabular}

(d) $\rho=0.9$

\begin{tabular}{cccc}
\hline 1509 & 896 & 328 & 30 \\
102 & 766 & 1075 & 341 \\
8 & 152 & 826 & 1183 \\
0 & 9 & 160 & 2615 \\
\hline
\end{tabular}

From Tables 11(a) and 11(b), we can see that the value of $\hat{\Phi}_{S S}^{(\lambda)}$ (for an arbitrary fixed $\lambda$ ) increases as $\rho$ increases for each of Tables 9 and 10. Also, for any fixed $\rho$ (and for any $\lambda$ ), we can see that the value of $\hat{\Phi}_{S S}^{(\lambda)}$ is greater for Table $9\left(\mu_{2}-\mu_{1}=0.6\right)$ than for Table $10\left(\mu_{2}-\mu_{1}=0.4\right)$. 
Table 11. Estimate of $\Phi_{S S}^{(\lambda)}$ applied to Tables 9 and 10

(a) For Table 9

\begin{tabular}{rcccc}
\hline \multicolumn{1}{c}{$\lambda$} & $\rho=0$ & $\rho=0.3$ & $\rho=0.6$ & $\rho=0.9$ \\
\hline-0.4 & 0.081 & 0.127 & 0.226 & 0.675 \\
0.0 & 0.115 & 0.178 & 0.308 & 0.796 \\
0.6 & 0.146 & 0.222 & 0.372 & 0.858 \\
1.0 & 0.155 & 0.235 & 0.391 & 0.871 \\
1.6 & 0.158 & 0.239 & 0.396 & 0.874 \\
2.0 & 0.155 & 0.235 & 0.391 & 0.871 \\
\hline
\end{tabular}

(b) For Table 10

\begin{tabular}{rcccc}
\hline \multicolumn{1}{l}{$\lambda$} & $\rho=0$ & $\rho=0.3$ & $\rho=0.6$ & $\rho=0.9$ \\
\hline-0.4 & 0.035 & 0.060 & 0.108 & 0.421 \\
0.0 & 0.051 & 0.086 & 0.153 & 0.541 \\
0.6 & 0.065 & 0.108 & 0.191 & 0.622 \\
1.0 & 0.070 & 0.116 & 0.203 & 0.643 \\
1.6 & 0.071 & 0.118 & 0.207 & 0.648 \\
2.0 & 0.070 & 0.116 & 0.203 & 0.643 \\
\hline
\end{tabular}

For the square contingency table with underlying bivariate normal distribution with the equality of two variances, when $\mu_{1}<\mu_{2}$, the observations in the cells of lower left triangle of table tend to decreases as $\rho$ approaches 1 or as the difference between $\mu_{1}$ and $\mu_{2}$ increases (see Tables 9 and 10). [We note that when $\mu_{1}>\mu_{2}$, the observations in the cells of upper right triangle of table tends to decreases as $\rho$ approaches 1 or as the difference between $\mu_{1}$ and $\mu_{2}$ increases.] Thus, it would be natural to consider that as $\rho$ approaches 1 or as the difference between $\mu_{1}$ and $\mu_{2}$ increases, the degree of departure from the SS model approaches the maximum. Therefore, $\hat{\Phi}_{S S}^{(\lambda)}$ may be appropriate for measuring the degree of departure from SS for the square contingency table formed from the underlying bivariate normal distribution with the equality of two variances. 


\section{Relationship Between the Measure and the Conditional Sum-Symmetry Model}

For the $r \times r$ table, the conditional SS (CSS) model (Yamamoto et al. [17]) is defined by

$$
p_{1(t)}=\Delta p_{2(t)} \text { for } t=3, \ldots, 2 r-1 \text {. }
$$

A special case of the CSS model obtained by putting $\Delta=1$ is the SS model. When the CSS model holds, the measure $\Phi_{S S}^{(\lambda)}$ can be expressed as a function of parameter $\Delta$;

$$
\Phi_{S S}^{(\lambda)}=\frac{1}{2^{\lambda}-1}\left[2^{\lambda}\left\{\left(\frac{\Delta}{\Delta+1}\right)^{\lambda+1}+\left(\frac{1}{\Delta+1}\right)^{\lambda+1}\right\}-1\right] \text {, for } \lambda>-1,
$$

and the value at $\lambda=0$ is taken to be the limit $\lambda \rightarrow 0$. Therefore, for any $\lambda$ fixed, $\Phi_{S S}^{(\lambda)}=0$ if and only if the SS model holds, i.e., value of $\Delta$ is equal to 1. Also, $\Phi_{S S}^{(\lambda)}$ increases as the value of $\Delta$ becomes more distant from 1 . Therefore, the measure $\Phi_{S S}^{(\lambda)}$ would be appropriate to measure the degree of departure from the SS model.

\section{Concluding Remarks}

The proposed measure $\Phi_{S S}^{(\lambda)}$ is useful for measuring the degree of departure from the SS model toward the maximum departure of SS, i.e., the structure of $p_{1(t)}=0$ or $p_{2(t)}=0$ for $t=3, \ldots, 2 r-1$. Also, this measure is always range between 0 and 1 , without depending on the diagonal proportions, the dimension $r$ and sample size $n$. Thus, the measure $\Phi_{S S}^{(\lambda)}$ may be useful for comparing the degrees of departure from SS in several tables. In addition, the measure $\Phi_{S S}^{(\lambda)}$ is weighted average of submeasures $\left\{\Phi_{S S(t)}^{(\lambda)}\right\}$, so $\Phi_{S S}^{(\lambda)}$ lies between the minimum value and maximum value of $\left\{\Phi_{S S(t)}^{(\lambda)}\right\}$. 
For analyzing the square contingency table by using the measure $\Phi_{S S}^{(\lambda)}$, the analyst may be interested in which value of $\lambda$ is preferred for a given table. However, it seems difficult to discuss this. For analyzing given table, it seems to be important that the analyst calculates the values of $\hat{\Phi}_{S S}^{(\lambda)}$ for various values of $\lambda$ and discusses the degree of departure from SS in terms of these $\hat{\Phi}_{S S}^{(\lambda)}$.

Finally, we note that the measure $\Phi_{S S}^{(\lambda)}$ is not invariant under arbitrary similar permutations of row and column categories. Therefore, the measure should be applied to square contingency tables with ordered categories.

\section{References}

[1] Y. M. M. Bishop, S. E. Fienberg and P. W. Holland, Discrete Multivariate Analysis: Theory and Practice, Cambridge, The MIT Press, 1975.

[2] A. H. Bowker, A test for symmetry in contingency tables, Journal of the American Statistical Association 43 (1948), 572-574.

[3] H. Caussinus, Contribution à l'analyse statistique des tableaux de corrélation, Annales de la Faculté des Sciences de l’Université de Toulouse 29 (1965), 77-182.

[4] Centers for Disease Control and Prevention; National Centers for Health Statistics; The National Health and Nutrition Examination Survey; 1999-2000 Data Documentation, Codebook, and Frequencies; Audiometry (1999-2000).

https://wwwn.cdc.gov/Nchs/Nhanes/1999-2000/AUX1.htm

[5] Centers for Disease Control and Prevention; National Centers for Health Statistics; The National Health and Nutrition Examination Survey; 2003-2004 Data Documentation, Codebook, and Frequencies; Audiometry (2003-2004). https://wwwn.cdc.gov/Nchs/Nhanes/2003-2004/AUX_C.htm

[6] Centers for Disease Control and Prevention; National Centers for Health Statistics; The National Health and Nutrition Examination Survey; Audiometry/Tympanometry Procedures Manual (2009).

http://www.cdc.gov/nchs/data/nhanes/nhanes_09_10/audiometry_09.pdf

[7] Centers for Disease Control and Prevention; National Centers for Health Statistics; The National Health and Nutrition Examination Survey; Muscle Strength Procedures Manual (2011).

http://www.cdc.gov/nchs/data/nhanes/nhanes_11_12/Muscle_Strength_Proc_Manual.pdf 
[8] Centers for Disease Control and Prevention; National Centers for Health Statistics; The National Health and Nutrition Examination Survey; 2011-2012 Data Documentation, Codebook, and Frequencies; Muscle Strength - Grip Test (2011-2012).

$$
\text { https://wwwn.cdc.gov/Nchs/Nhanes/2011-2012/MGX_G.htm }
$$

[9] Centers for Disease Control and Prevention; National Centers for Health Statistics; The National Health and Nutrition Examination Survey; 2011-2012 Data Documentation, Codebook, and Frequencies; Demographic Variables and Sample Weights (2011-2012).

https://wwwn.cdc.gov/Nchs/Nhanes/2011-2012/DEMO_G.htm

[10] N. Cressie and T. R. C. Read, Multinomial goodness-of-fit tests, Journal of the Royal Statistical Society, Series B 46 (1984), 440-464.

[11] G. P. Patil and C. Taillie, Diversity as a concept and its measurement, Journal of the American Statistical Association 77 (1982), 548-561.

[12] T. R. C. Read and N. Cressie, Goodness-of-Fit Statistics for Discrete Multivariate Data, New York, Springer, 1988.

[13] A. Stuart, A test for homogeneity of the marginal distributions in a two-way classification, Biometrika 42 (1955), 412-416.

[14] K. Tahata and S. Tomizawa, Symmetry and asymmetry models and decompositions of models for contingency tables, SUT Journal of Mathematics 50 (2014), 131-165.

[15] S. Tomizawa, Two kinds of measures of departure from symmetry in square contingency tables having nominal categories, Statistica Sinica 4 (1994), 325-334.

[16] S. Tomizawa, T. Seo and H. Yamamoto, Power-divergence-type measure of departure from symmetry for square contingency tables that have nominal categories, Journal of Applied Statistics 25 (1998), 387-398.

[17] K. Yamamoto, Y. Tanaka and S. Tomizawa, Sum-symmetry model and its orthogonal decomposition for square contingency tables with ordered categories, SUT Journal of Mathematics 49 (2013), 121-128. 